OSLO-TP 2-97

cond-mat/9701154

\title{
Statistical mechanics and thermodynamics for multispecies exclusion statistics
}

\author{
Serguei B. Isakov ${ }^{a, b}$, Stefan Mashkevich ${ }^{a, c}$ \\ ${ }^{a}$ Senter for Høyere Studier, Drammensveien 78, 0271 Oslo, Norway \\ ${ }^{b}$ Department of Physics, University of Oslo \\ P.O. Box 1048 Blindern, 0316 Oslo, Norway \\ ${ }^{c}$ Institute for Theoretical Physics, 252143 Kiev, Ukraine
}

\begin{abstract}
Statistical mechanics and thermodynamics for ideal fractional exclusion statistics with mutual statistical interactions is studied systematically. We discuss properties of the single-state partition functions and derive the general form of the cluster expansion. Assuming a certain scaling of the single-particle partition functions, relevant to systems of noninteracting particles with various dispersion laws, both in a box and in an external harmonic potential, we derive a unified form of the virial expansion. For the case of a symmetric statistics matrix at a constant density of states, the thermodynamics is analyzed completely. We solve the microscopic problem of multispecies anyons in the lowest Landau level for arbitrary values of particle charges and masses (but the same sign of charges). Based on this, we derive the equation of state which has the form implied by exclusion statistics, with the statistics matrix coinciding with the exchange statistics matrix of anyons. Relation to one-dimensional integrable models is discussed.
\end{abstract}

Keywords: exclusion statistics, equation of state, harmonic potential, CalogeroSutherland model, anyons, lowest Landau level

\footnotetext{
${ }^{1}$ Present address. Email: serguei.isakov@phys.uio.no

${ }^{2}$ Present address. Email: mash@phys.ntnu.no
} 


\section{Introduction}

In recent years it has been appreciated that there exists a nontrivial possibility of mutual quantum statistics, or "statistical interaction" between distinguishable particles. One example is multispecies exclusion statistics [1], which postulates that inserting into the system a particle of species $b$ reduces the number $D_{a}$ of single-particle states available for species $a$ by some $g_{a b}$. The quantities $g_{a b}$ constitute the exclusion statistics matrix (later referred to as the statistics matrix) and are called mutual statistics parameters for $a \neq b$.

Another example is multispecies exchange statistics, or multispecies anyons [2, 3, 4. Their definition is the following: (i) when two particles $a$ are interchanged, the two-body wave function acquires a phase factor $\exp \left[\mathrm{i} \pi \alpha_{a a}\right]$; (ii) when particle $a$ encircles particle $b$, it acquires a phase factor $\exp \left[2 \mathrm{i} \pi \alpha_{a b}\right]$. The matrix $\left\{\alpha_{a b}\right\}$ will be called exchange statistics matrix.

In fact, there is a connection between the two models, which was first established for a single species: The thermodynamic quantities for a system of anyons (in a box) confined to the lowest Landau level (LLL) of a strong external magnetic field [5] are the same as those of exclusion statistics particles, all having the same energy (energy of the LLL) upon identification $g=\alpha$ [6]. This correspondence was extended to the multispecies model in the particular case when the electric charges of all the species are equal; one then has $g_{a b}=\alpha_{a b}$ 《4.

The relation between the two models was also discussed in the context of an algebraic approach to statistics (aimed at searching for an algebra of observables for identical particles). The algebraic definition of fractional statistics in one dimension [7] applies to anyons in the LLL, where the dynamics is effectively one-dimensional [8]. On the other hand, this definition suggests a realization of 1D fractional statistics by systems with inverse square interactions [7] (see also [9]), which has been justified by interpreting the thermodynamics of the Calogero and Sutherland models [10, 11] as that of systems of noninteracting particles [12]. The statistical distribution for $1 \mathrm{D}$ fractional statistics found in this way [12] turns out to be the same as that derived from Haldane's multiplicity formula for exclusion statistics [13, 6].

In addition to the above, (single-species) integrable models of the CalogeroSutherland type were directly related to exclusion statistics [14, 15, 16]. It is therefore natural to expect that there should exist multispecies generalizations of those models that would correspond to multispecies exclusion statistics.

It is generally believed that excitations in the fractional quantum Hall effect (FQHE) can be described either as anyons (by a Berry phase argument [17]) or as exclusion statistics particles (by a state-counting argument [1, 18]). In particular, when excitations with charges of different magnitudes are present - in multilayer systems [3] and in the hierarchical structure [19] - the multispecies model is applicable. Another problem of interest is that of FQHE quasielectrons and quasiholes, again viewed as two species. By counting the states, their off-diagonal exclusion statistics parameters were recently shown [20] to be antisymmetric; on 
the other hand, the exchange statistics matrix is always symmetric by definition. How the two descriptions match in this case, is not clear by now.

Therefore, developing statistical mechanics for mutual statistics is of interest both theoretically and phenomenologically. Thus far, some progress has been achieved on the issue [13, 21, 14, generalizing the results of the better-understood single-species case, where the thermodynamics was studied for a system in a box [6, 15, 22, 23] as well as in a harmonic potential [15, 24, 25].

This paper is devoted to systematically studying statistical mechanics of particles of arbitrary number of species obeying exclusion statistics and calculating their thermodynamic quantities.

In Sec. 2 we introduce the single-state grand canonical partition function and prove that it factorizes into a product of partition functions corresponding to separate species. We infer a general formula for the coefficients of the expansion of its associated single-state thermodynamic potential in powers of the single-state Gibbs factors. Those coefficients, multiplied by dimensional factors depending on the dispersion law of the particles and the dimension of space, give the cluster coefficients, which are discussed in Sec. 3. Assuming a certain temperature scaling of the single-particle partition functions — which holds for systems of noninteracting particles with various dispersion laws, both in a box and in an external harmonic potential, - we also derive a unified form of the virial expansion, calculating explicitly a few first coefficients.

Section 4 is devoted to a specific case when the statistics matrix is symmetric and in addition the density of single-particle states is constant in energy. This case can be analyzed completely, yielding a closed form of the equation of state, which may be expanded to give both low-temperature and low-density series. In Sec. 5 we discuss various physical examples of exclusion statistics for systems of noninteracting particles both in a box and in an external harmonic potential.

In Sec. 6 we address the problem of multispecies anyons in the LLL at different values (but the same sign) of charges and different masses (hence different cyclotron frequencies) for different species. In Subsec. 6.1 we solve this problem exactly, exposing anyons of different species to harmonic potentials with different frequencies such that the resulting level spacing is the same for all the species. In Subsec. 6.2 we derive the equations of state for multispecies anyons in the LLL both in a harmonic potential and in a box. It has the form implied by exclusion statistics, with the statistics matrix coinciding with the exchange statistics matrix of anyons. Relation to exclusion statistics is also demonstrated by a semiclassical argument in Subsec. 6.3.

We conclude with remarks on searching for microscopic integrable models for multispecies exclusion statistics. 


\section{Partition functions}

The definition of exclusion statistics is the following [1]. If $G_{a}$ is the initial singleparticle Hilbert space dimension for species $a$ and $N_{a}$ the number of particles of that species, then (i) the reduced Hilbert space dimension is

$$
D_{a}=G_{a}-\sum_{b} g_{a b}\left(N_{b}-\delta_{a b}\right)
$$

and (ii) the many-particle statistical weights (multiplicities) are determined as

$$
W=\prod_{a} \frac{\left(D_{a}+N_{a}-1\right) !}{N_{a} !\left(D_{a}-1\right) !} .
$$

Applying (2.1)-(2.2) locally in phase space, so that the total multiplicity is

$$
W=\prod_{i} W^{(i)}
$$

where $i$ labels groups of single-particle states of nearby energy $\varepsilon^{(i)}$, and $W^{(i)}$ is given by (2.2) with the changes $N_{a} \rightarrow N_{a}^{(i)}, G_{a} \rightarrow G_{a}^{(i)}$, and $D_{a} \rightarrow D_{a}^{(i)}$, makes the definition of ideal fractional exclusion statistics for many species [13, 6] (see also a related discussion of systems with internal degrees of freedom [26]).

The above equations make the starting point for constructing statistical mechanics. Assuming that $G_{a}^{(i)}$ does not depend on $a$. 5 one deduces equations for the average occupation numbers $n_{a}^{(i)}=N_{a}^{(i)} / G_{a}^{(i)}$ in the thermodynamic limit, which can be conveniently written as 13, 6

$$
\frac{w_{a}^{(i)}}{\prod_{b}\left(1-w_{b}^{(i)}\right)^{g_{b a}}}=x_{a}^{(i)},
$$

where $x_{a}^{(i)}=e^{\beta\left(\mu_{a}-\varepsilon_{a}^{(i)}\right)}$ and

$$
w_{a}^{(i)}=\frac{n_{a}^{(i)}}{1+n_{a}^{(i)}-\sum_{b} g_{a b} n_{b}^{(i)}} .
$$

It is natural to introduce the single-state grand canonical partition function $\xi^{(i)}$, which determines the occupation numbers as [13]

$$
n_{a}^{(i)}=x_{a}^{(i)} \frac{\partial}{\partial x_{a}^{(i)}} \ln \xi^{(i)} .
$$

It was observed [4] that for a symmetric statistics matrix, $g_{a b}=g_{b a}$, the partition function $\xi^{(i)}$ factorizes as

$$
\xi^{(i)}=\prod_{a} \xi_{a}^{(i)}
$$

\footnotetext{
${ }^{3}$ This holds for a wide class of practically interesting systems (see below, Sec. 同). However, the case of $G_{a}^{(i)}$ being $a$ dependent could be worked out as well; see, e.g., Eqs. (6.16)- 6.18) and the discussion thereafter.
} 
where the grand partition function $\xi_{a}^{(i)}$ for particles of species $a$ is connected with the occupation numbers by [see (2.5)]

$$
\xi_{a}^{(i)}=\frac{1}{1-w_{a}^{(i)}}
$$

The relations (2.5)-(2.8) were found in Ref. [4 by explicitly expanding (for the case of two species) the functions $\xi_{a}^{(i)}, \xi^{(i)}$, and $n_{a}^{(i)}$ in powers of the Gibbs factors $\left\{x_{a}^{(i)}\right\}$. We observe here that the above relations can be put on a more general ground. Namely, in the generic case of a nonsymmetric $g_{a b}$, from Eq. (2.4) we find [we drop the superscript $(i)$ from now on]

$$
\frac{\partial w_{a}}{\partial x_{b}}=\frac{1}{x_{b}}\left(D^{-1}\right)_{a b}
$$

where

$$
D_{a b}=\frac{\delta_{a b}}{w_{a}}+\frac{g_{b a}}{1-w_{b}}
$$

Equations (2.6)-(2.8) then imply that $D_{a b}$ relates the partition functions to the occupation numbers:

$$
\sum_{a} n_{a} D_{a b}=\xi_{b}
$$

Substituting (2.8) and (2.10) herein recovers (2.5). This shows that (2.6)-(2.8) are consistent with (2.4), 2.5) in the generic case (any dispersion law and a nonsymmetric matrix $\left.g_{a b}\right)$.

Equations (2.4), (2.7), and (2.8) implicitly determine $\xi$ as a function of the Gibbs factors $\left\{x_{a}\right\}$. In particular, expanding

$$
\ln \xi=\sum_{k_{1} \ldots k_{s}} f_{k_{1} \ldots k_{s}} x_{1}^{k_{1}} \cdots x_{s}^{k_{s}}
$$

where $s$ is the number of species, one can find the coefficients $f_{k_{1} \ldots k_{s}}$ one by one. We infer a general formula based on explicit calculations for low orders,

$$
f_{k_{1} \ldots k_{r} \underbrace{0 \ldots 0}_{s-r}}=(-1)^{r-1} \frac{\prod_{j=1}^{r} \prod_{l=1}^{k_{j}-1}\left(1-\frac{\sum_{n=1}^{r} k_{n} g_{j n}}{l}\right)}{\prod_{n=1}^{r} k_{n}} \mathcal{F}_{k_{1} \ldots k_{r}},
$$

where

$$
\mathcal{F}_{k_{1} \ldots k_{r}}=\sum_{\substack{p_{1}, q_{1}, \ldots, p_{r}-1, q_{r-1}=1}}^{r} *\left(\prod_{n=1}^{r-1} k_{q_{n}} g_{p_{n} q_{n}}\right)
$$

Here all the numbers $k_{1}, \ldots, k_{r}$ are assumed to be different from zero, and the $p_{n}$ 's and $q_{n}$ 's summed over in (2.14) are constrained as follows: (i) $p_{1}<p_{2}<$ 
$\ldots<p_{r-1}$; (ii) $p_{n} \neq q_{n}$; (iii) it may not be $p_{m}=q_{n}$ and $q_{m}=p_{n}$; (iv) at least one of the $q_{n}$ 's has to be equal to the number that is lacking in the set $\left\{p_{1}, \ldots, p_{r-1}\right\}$.

For example:

$$
\begin{aligned}
\mathcal{F}_{k_{1}} & =1 \\
\mathcal{F}_{k_{1} k_{2}} & =k_{1} g_{21}+k_{2} g_{12} \\
\mathcal{F}_{k_{1} k_{2} k_{3}} & =k_{1}^{2} g_{21} g_{31}+k_{1} k_{2} g_{21} g_{32}+k_{3} k_{1} g_{23} g_{31}+\text { cycl. perm. }
\end{aligned}
$$

where "cycl. perm." denotes terms obtained by simultaneous cyclic permutations of the subscripts of $g$ 's and $k$ 's. Hence one gets

$$
f_{k 0 \ldots 0}=\frac{1}{k} \prod_{l=1}^{k-1}\left(1-\frac{k g_{11}}{l}\right)
$$

(cf. [12]) as well as

$$
\begin{aligned}
f_{110 \ldots 0} & =-\left(g_{12}+g_{21}\right) ; \\
f_{210 \ldots 0} & =-\left(g_{12}+2 g_{21}\right)\left(1-2 g_{11}-g_{12}\right) / 2, \\
f_{1110 \ldots 0} & =g_{21} g_{31}+g_{21} g_{32}+g_{23} g_{31}+\text { cycl. perm. . }
\end{aligned}
$$

For two species and a symmetric matrix $g_{a b}$, the coefficients (2.13) reduce to those obtained in Ref. [1].

One can also evaluate the coefficients of the expansions

$$
\ln \xi_{a}=\sum_{k_{1} \ldots k_{s}} f_{k_{1} \ldots k_{s}}^{a} x_{1}^{k_{1}} \cdots x_{s}^{k_{s}} ;
$$

the answer is that $f_{k_{1} \ldots k_{s}}^{a}$ is obtained from (2.13) by replacing $\mathcal{F}_{k_{1} \ldots k_{r}}$ with $\mathcal{F}_{k_{1} \ldots k_{r}}^{a}$, which in turn is obtained from (2.14) by restricting that $p_{n} \neq a$ for any $n$ (thereby fixing all the $p_{n}$ 's). For example,

$$
\begin{aligned}
\mathcal{F}_{k_{1} k_{2}}^{1} & =k_{1} g_{21}, \quad \mathcal{F}_{k_{1} k_{2}}^{2}=k_{2} g_{12} ; \\
\mathcal{F}_{k_{1} k_{2} k_{3}}^{1} & =k_{1}^{2} g_{21} g_{31}+k_{1} k_{2} g_{21} g_{32}+k_{3} k_{1} g_{23} g_{31}, \quad \text { etc. }
\end{aligned}
$$

For a symmetric matrix $g_{a b}$, we observe an important property

$$
f_{k_{1} \ldots k_{s}}^{a}=\frac{k_{a}}{k_{1}+\cdots+k_{s}} f_{k_{1} \ldots k_{s}},
$$

which will be used in Sec. 1 when deriving an equation of state.

\section{Cluster and virial expansions}

The formulas of the previous section enable one to compute the equation of state provided the single-particle spectrum is known. Let the latter be $\varepsilon_{a}^{(i)}=\varepsilon_{a}^{(0)}+\zeta^{(i)}$, 
where the $\zeta^{(i)}$ 's are common for all the species. By summing 2.12) over singleparticle states $\left(\sum_{i} \ln \xi^{(i)}=\ln \Xi=-\beta \Omega\right)$, one then obtains a cluster expansion

$$
-\beta \Omega=\sum_{k_{1} \ldots k_{s}} b_{k_{1} \ldots k_{s}} z_{1}^{k_{1}} \cdots z_{s}^{k_{s}}, \quad z_{a}=e^{\beta \mu_{a}}
$$

with the cluster coefficients

$$
b_{k_{1} \ldots k_{s}}=Z_{1}^{\prime}(K \beta) e^{-\beta \sum_{a} k_{a} \varepsilon_{a}^{(0)}} f_{k_{1} \ldots k_{s}}
$$

where $K=\sum_{n=1}^{s} k_{n}$ and

$$
Z_{1}^{\prime}(\beta)=\sum_{i} e^{-\beta \zeta^{(i)}}
$$

is the (species-independent) "shifted" single-particle partition function, corresponding to counting the single-particle energy from the ground state. One has $Z_{1}^{a}(\beta) \equiv \sum_{i} e^{-\beta \varepsilon_{a}^{(i)}}=e^{-\beta \varepsilon_{a}^{(0)}} Z_{1}^{\prime}(\beta)$. Assume that $Z_{1}^{\prime}(\beta)$ scales with the inverse temperature $\beta$ as (cf. 25)

$$
Z_{1}^{\prime}(K \beta)=\frac{Z_{1}^{\prime}(\beta)}{K^{1+\delta}}
$$

We will see below that this scaling is relevant in the thermodynamic limit for various physical systems. The cluster coefficients (3.2) then become

$$
b_{k_{1} \ldots k_{s}}=\frac{Z_{1}^{\prime}(\beta)}{K^{1+\delta}} e^{-\beta \sum_{a} k_{a} \varepsilon_{a}^{(0)}} f_{k_{1} \ldots k_{s}} .
$$

The expression for particle numbers $N_{a}=z_{a} \frac{\partial}{\partial z_{a}} \ln \Xi$ reads

$$
N_{a}=\sum_{k_{1} \ldots k_{s}} k_{a} b_{k_{1} \ldots k_{s}} z_{1}^{k_{1}} \cdots z_{s}^{k_{s}}
$$

Using (2.13) to determine the cluster coefficients (3.5), one then deduces from (3.1) and (3.6) the "virial expansion"

$$
-\beta \Omega=\sum_{k_{1} \ldots k_{s}} A_{k_{1} \ldots k_{s}} \frac{N_{1}^{k_{1}} \cdots N_{s}^{k_{s}}}{\left[Z_{1}^{\prime}(\beta)\right]^{k_{1}+\cdots+k_{s}-1}},
$$

where the dimensionless "virial coefficients" $A_{k_{1} \ldots k_{s}}$ can all be evaluated exactlyt, even if there is apparently no general expression for them. Up to the third order,

\footnotetext{
${ }^{4}$ Expressions for virial coefficients in terms of cluster coefficients for two species may be found in Ref. 何. We give here the simplest formula of that kind for three species: $A_{111}=$ $2 \tilde{b}_{011} \tilde{b}_{101}+2 \tilde{b}_{011} \tilde{b}_{110}+2 \tilde{b}_{101} \tilde{b}_{110}-2 \tilde{b}_{111}$, where $\tilde{b}_{k_{1} k_{2} k_{3}}=b_{k_{1} k_{2} k_{3}} / b_{100}^{k_{1}} b_{010}^{k_{2}} b_{001}^{k_{3}}$, as well as the associated formula relating the cluster coefficient to many-particle partition functions: $b_{111}=$ $2 Z_{001} Z_{010} Z_{100}-Z_{100} Z_{011}-Z_{010} Z_{101}-Z_{001} Z_{110}+Z_{111}$.
} 
they are:

$$
\begin{aligned}
A_{10 \ldots 0}= & 1 ; \\
A_{20 \ldots 0}= & -2^{-2-\delta}\left(1-2 g_{11}\right), \\
A_{110 \ldots 0}= & 2^{-1-\delta}\left(g_{12}+g_{21}\right) ; \\
A_{30 \ldots 0}= & \left(4^{-1-\delta}-2 \cdot 3^{-2-\delta}\right)-\left(4^{-\delta}-3^{-\delta}\right) g_{11}\left(1-g_{11}\right), \\
A_{210 \ldots 0}= & -4^{-1-\delta}\left(g_{12}+g_{21}\right)\left(2-4 g_{11}-g_{12}-g_{21}\right) \\
& +3^{-1-\delta}\left(g_{12}+2 g_{21}\right)\left(1-2 g_{11}-g_{12}\right), \\
A_{1110 \ldots 0}= & -2 \cdot 3^{-1-\delta}\left(g_{21} g_{31}+g_{21} g_{32}+g_{23} g_{31}\right) \\
& +4^{-1 / 2-\delta}\left(g_{21} g_{31}+g_{21} g_{32}+g_{23} g_{31}+g_{12} g_{13}\right) \\
& + \text { cycl. perm. }
\end{aligned}
$$

Note that the mixed virial coefficient of order two vanishes for an antisymmetric statistics matrix.

For a symmetric statistics matrix, the last two expressions become

$$
\begin{aligned}
A_{210 \ldots 0} & =-\left(4^{-\delta}-3^{-\delta}\right) g_{12}\left(1-2 g_{11}-g_{12}\right), \\
A_{1110 \ldots 0} & =2\left(4^{-\delta}-3^{-\delta}\right)\left(g_{12} g_{13}+g_{12} g_{23}+g_{13} g_{23}\right) .
\end{aligned}
$$

If in addition $\delta=0$, then the virial coefficients of total order three do not depend on the statistics parameters. This case will be discussed in detail in the next section.

\section{Thermodynamics for a symmetric statistics matrix at a constant density of states}

The case of $g_{a b}=g_{b a}$ and $\delta=0$, in fact, can be completely analyzed. It is also of particular interest since it means a $1 / \beta$ scaling for $Z_{1}^{\prime}(\beta)$, i.e., a constant density of states in energy. The particle numbers (3.6) read

$$
N_{a}=Z_{1}^{\prime}(\beta) \sum_{k_{1} \ldots k_{s}} \frac{k_{a}}{k_{1}+\cdots+k_{s}} f_{k_{1} \ldots k_{s}} z_{1}^{k_{1}} \cdots z_{s}^{k_{s}} .
$$

The statistics matrix being symmetric, using Eq. (2.20) yields an important relation

$$
N_{a}=\left.Z_{1}^{\prime}(\beta) \ln \xi_{a}\right|_{x_{b} \rightarrow z_{b}} \text { for all } b,
$$

which enables one to use all the formulas from the previous section provided the arguments of the functions $\xi_{a}$ and $w_{a}$, the Gibbs factors $\left\{x_{b}\right\}$, are replaced by the fugacities $\left\{z_{b}\right\}$.

To deduce the equation of state, consider the derivatives

$$
-\frac{\partial \beta \Omega}{\partial N_{a}}=\sum_{b} \frac{\partial z_{b}}{\partial N_{a}} \frac{N_{b}}{z_{b}} .
$$


From (4.2) we find

$$
\frac{\partial z_{b}}{\partial N_{a}}=\frac{\left(M^{-1}\right)_{b a}}{Z_{1}^{\prime}(\beta)}
$$

where the matrix $M_{a b} \equiv \frac{\partial}{\partial z_{b}} \ln \xi_{a}$ can be expressed, with the use of (2.8) and (2.9), as

$$
M_{a b}=\frac{\xi_{a}}{z_{b}}\left(D^{-1}\right)_{a b}
$$

and consequently

$$
\left(M^{-1}\right)_{b a}=\frac{z_{b}}{\xi_{a}} D_{b a}
$$

Inserting this into (4.4) and then the result into (4.3), we obtain

$$
-\frac{\partial \beta \Omega}{\partial N_{a}}=\frac{N_{a}}{Z_{1}^{\prime}(\beta)} \frac{1}{e^{N_{a} / Z_{1}^{\prime}(\beta)}-1}+\sum_{b} g_{a b} \frac{N_{b}}{Z_{1}^{\prime}(\beta)},
$$

where the symmetry of the statistics matrix was used. Integrating (4.7) yields finally the equation of state

$$
-\Omega=\frac{1}{\beta Z_{1}^{\prime}(\beta)}\left\{\sum_{a} \int_{0}^{N_{a}} \frac{\zeta_{a} d \zeta_{a}}{e^{\zeta_{a} / Z_{1}^{\prime}(\beta)}-1}+\frac{1}{2} \sum_{a b} g_{a b} N_{a} N_{b}\right\} .
$$

Due to the scaling $Z_{1}^{\prime}(\beta) \propto \beta^{-1}$, only zero-temperature terms in the last expression depend on the statistics parameters. Equation (4.8) can be expanded to give both low-temperature and low-density (high-temperature) series. In the former case, restricting to the perturbative (behaving as powers of the temperature) terms, one obtains

$$
-\Omega=E=\frac{\pi^{2}}{6} s Z_{0} T^{2}+\frac{1}{2 Z_{0}} \sum_{a b} g_{a b} N_{a} N_{b}, \quad Z_{0} \equiv \beta Z_{1}^{\prime}(\beta) .
$$

This in particular implies that the specific heat is $C=\frac{1}{3} \pi^{2} s Z_{0} T$ to all orders in $T$, which is the same as that for a mixture of Fermi gases (one should impose the condition $g>0$ to do the low-temperature expansion).

In the opposite limit, for small $N_{a} / Z_{1}^{\prime}(\beta)$, one can expand (4.8) to obtain

$$
-\beta \Omega=\sum_{a} N_{a}+\frac{1}{2} \sum_{a b}\left(g_{a b}-\frac{1}{2} \delta_{a b}\right) \frac{N_{a} N_{b}}{Z_{1}^{\prime}(\beta)}+\sum_{a} \sum_{k=2}^{\infty} \frac{\mathcal{B}_{k}}{(k+1) !}\left(\frac{N_{a}}{Z_{1}^{\prime}(\beta)}\right)^{k+1}
$$

where $\mathcal{B}_{k}$ are the Bernoulli numbers $\left(\mathcal{B}_{2}=\frac{1}{6}, \mathcal{B}_{4}=-\frac{1}{30}\right.$, etc., $\left.\mathcal{B}_{2 l+1}=0\right)$. Thus, just as Eq. (3.8) shows, it is only the second order virial coefficients that depend on the statistics matrix. Eqs. (4.8)-(4.10) are generalizations of the results of Refs. [15, 24, 23, 25] to the multispecies case. 


\section{Examples}

In this section we consider particular dispersion laws of particles, mostly discussing examples which are related to integrable models. For a single species, it has been shown that integrable models with inverse square interactions, the Calogero [10] and Sutherland [11] models, are equivalent thermodynamically to systems of noninteracting particles obeying exclusion statistics. This naturally suggests that there should exist generalizations of those models corresponding to multispecies exclusion statistics. It is in this sense that we refer below to the "multispecies Calogero and Sutherland models" (which have yet to be discovered). The equations of state found below for noninteracting particles obeying exclusion statistics apply at the same time to the conjectured multispecies integrable models, thus providing guidelines for their search.

\subsection{Particles in a box}

Consider a gas of free particles in a $D$-dimensional box of volume $V$. In the quasicontinuous spectrum approximation, when the momentum summation can be replaced by integration, for particles with the dispersion law $\varepsilon_{a}(p)=\varepsilon_{a}^{(0)}+\Lambda p^{\sigma}$ $(\sigma \neq 0)$ the shifted single-particle partition function reads 23,25

$$
Z_{1}^{\prime}(\beta)=\frac{\Gamma(1+D / \sigma) V}{(2 \sqrt{\pi})^{D} \Gamma(1+D / 2)(\Lambda \beta)^{D / \sigma}}
$$

satisfying (3.4) with $\delta=D / \sigma-1$. One has $\Omega=-P V$, where $P$ is the pressure, and the expansion (3.7) becomes the usual virial expansion for a system in a box:

$$
\beta P=\sum_{k_{1} \ldots k_{s}} a_{k_{1} \ldots k_{s}} \rho_{1}^{k_{1}} \cdots \rho_{s}^{k_{s}}, \quad \rho_{a}=N_{a} / V
$$

where the (dimensional) virial coefficients are

$$
a_{k_{1} \ldots k_{s}}=A_{k_{1} \ldots k_{s}}\left(\frac{V}{Z_{1}^{\prime}(\beta)}\right)^{k_{1}+\cdots+k_{s}-1}
$$

with $A_{k_{1} \ldots k_{s}}$ defined in Sec. 3 .

\subsection{1 "Multispecies Sutherland model" ( $D=1, \sigma=2, \delta=-1 / 2)$}

For a single species of particles with a quadratic dispersion law $\varepsilon_{a}(p)=p^{2} / 2 m$ in one dimension, it is the Sutherland model that realizes exclusion statistics. For a conjectured multispecies version of that model, the dimensional factor in (5.3) relating the dimensional virial coefficients to the dimensionless ones is $V / Z_{1}^{\prime}(\beta)=\lambda_{T}$, where $\lambda_{T}=\sqrt{2 \pi \beta / m}$ is the thermal wavelength. The singlespecies expressions agree with those obtained in Refs. 227, 23. 


\subsubsection{Chiral fields on a circle $(D=1, \sigma=1, \delta=0)$}

The model of chiral fields on a circle [28] (a generalization of the model [29] to the multispecies case) is a field theoretical realization of exclusion statistics, with a symmetric statistics matrix, for particles with linear dispersion (above a gap) propagating in only one direction: $\varepsilon_{a}(p)=\varepsilon_{a}^{(0)}+v p, p \geq 0$. Due to the latter condition, $Z_{1}^{\prime}(\beta)=V / 2 \pi v \beta$, half of the value given by (5.1). Since $\delta=0$, the equation of state is given by (4.8); the low-temperature expansion (4.9) agrees with that found in Ref. [28.

\subsubsection{Nonrelativistic particles in 2 dimensions $(D=2, \sigma=2, \delta=0)$}

Particles with a quadratic dispersion law in two dimensions and a symmetric statistics matrix (a conjectured model of Ref. [30], see Sec. 7) also fall into the category considered in Sec. 4 so that the equation of state is again (4.8), with $V / Z_{1}^{\prime}(\beta)=\lambda_{T}^{2}$.

\subsection{Particles in a harmonic potential}

We now consider systems of noninteracting nonrelativistic particles in a harmonic well of strength $\omega$ in $D$ dimensions. The single-particle partition function is

$$
Z_{1}^{a}(\beta)=\frac{e^{-\frac{1}{2} D \beta \omega}}{\left(1-e^{-\beta \omega}\right)^{D}} .
$$

Expanding this to the leading order in $\beta \omega$ results in

$$
Z_{1}^{\prime}(\beta) \simeq \frac{1}{(\beta \omega)^{D}}
$$

thus respecting the scaling (3.4) with $\delta=D-1$ (with $D=1$ as the condition for the density of states to be constant). This leads to the equation of state (3.7) [and to (4.8) for $D=1$ and a symmetric statistics matrix].

If one considers the correction terms, of order $\mathcal{O}(1)$ in (5.5), they might lead to corrections to the virial coefficients of very high order, $N$ and above. However, if the virial expansion converges, these corrections are negligible inside the radius of convergence.

Note that for particles in a harmonic potential, there is no well defined volume occupied by the gas; therefore, the equation of state is understood as an equation relating the thermodynamic potential $\Omega$ to the particle numbers, temperature, and the harmonic frequency $\omega$. Note also that the single-particle dispersion law is linear $(\sigma=1)$ here, because the single-particle energy depends linearly on the quantum numbers. 


\subsection{1 "Multispecies Calogero model" ( $D=1, \sigma=1, \delta=0)$}

For a single species with a linear dispersion law, the Calogero model realizes exclusion statistics. The "multispecies Calogero model", which would be equivalent to a system of noninteracting particles with a symmetric statistics matrix in a one-dimensional harmonic potential [30], would again fall into the class considered in Sec. 4 and be governed by the equation of state (4.8), with $Z_{1}^{\prime}(\beta)=1 / \beta \omega$.

\subsubsection{Harmonic potential in 2 dimensions $(D=2, \sigma=1, \delta=1)$}

For the same model as in 5.1 .3 but involving a harmonic well, i.e., a linear dispersion law, whence $Z_{1}^{\prime}(\beta)=1 /(\beta \omega)^{2}$, the equation of state is the generic Eq. (3.7) with the "virial coefficients" (3.8).

Another important example, to be considered in detail in the next section, is multispecies anyons in the LLL, both in a harmonic potential and in a box.

\section{Multispecies anyons in the lowest Landau level}

The problem of anyons in the LLL, being of relevance, in particular, to the FQHE, was originally solved for a single species in Ref. [5]. The multispecies version of the problem was addressed in Ref. [1], with the restriction that all the species have the same electric charge and mass, and the equation of state in a box was derived. Here we revisit this problem, allowing anyons of different species to have different magnitudes (but the same sign) of charges and different masses. We derive the equations of state in a box and in a harmonic potential, showing how they fit into our general framework, and provide a simple semiclassical picture.

\subsection{Spectrum in a harmonic potential}

The single-particle Hamiltonian in a magnetic field and a harmonic potential is

$$
H=-\frac{2}{m} \partial \bar{\partial}-\omega_{\mathrm{c}}(z \partial-\bar{z} \bar{\partial})+\frac{m \omega_{\mathrm{t}}^{2}}{2} z \bar{z}
$$

$(z=x+\mathrm{i} y, \partial=\partial / \partial z, \bar{\partial}=\partial / \partial \bar{z})$, and its spectrum is

$$
E_{\ell n}=\left(\ell+\frac{1}{2}\right)\left(\omega_{\mathrm{t}}-\omega_{\mathrm{c}}\right)+\left(n+\frac{1}{2}\right)\left(\omega_{\mathrm{t}}+\omega_{\mathrm{c}}\right)
$$

where $\omega_{\mathrm{c}}=-e B / 2 m(e B<0), \omega_{\mathrm{t}}=\sqrt{\omega_{\mathrm{c}}^{2}+\omega^{2}}$, with $\ell, n=0,1,2, \ldots$ The quantum numbers $\ell$ and $n$ are, respectively, the angular momentum and the number of the Landau level. The LLL restriction, $n=0$, leads to

$$
E_{\ell}=\omega_{\mathrm{t}}+\ell \varpi
$$

with $\varpi=\omega_{\mathrm{t}}-\omega_{\mathrm{c}}$. 
In the multispecies problem, the charges $e_{a}$ and the masses $m_{a}$ being different, so are the cyclotron frequencies $\omega_{c a}$. Let the harmonic frequencies $\omega_{a}$ be different as well. The many-body Hamiltonian becomes

$$
H_{N}=\sum_{a j}\left[-\frac{2}{m_{a}} \partial_{a j} \bar{\partial}_{a j}-\omega_{\mathrm{ca}}\left(z_{a j} \partial_{a j}-\bar{z}_{a j} \bar{\partial}_{a j}\right)+\frac{m_{a} \omega_{\mathrm{ta}}^{2}}{2} z_{a j} \bar{z}_{a j}\right]
$$

( $j$ numbers particles of a given species, $\partial_{a j}=\partial / \partial z_{a j}$ ). Making an ansatz

$$
\Psi_{N}=\tilde{\Psi}_{N} \exp \left(-\sum_{a j} \frac{m_{a} \omega_{\mathrm{t} a}}{2} z_{a j} \bar{z}_{a j}\right)
$$

yields the Hamiltonian acting on $\tilde{\Psi}_{N}$,

$$
\tilde{H}_{N}=\sum_{a j}\left[-\frac{2}{m_{a}} \partial_{a j} \bar{\partial}_{a j}+\left(\omega_{\mathrm{t} a}-\omega_{\mathrm{c} a}\right) z_{a j} \partial_{a j}+\left(\omega_{\mathrm{t} a}+\omega_{\mathrm{c} a}\right) \bar{z}_{a j} \bar{\partial}_{a j}+\omega_{\mathrm{t} a}\right] .
$$

We now choose the $\omega_{a}$ 's such that $\varpi \equiv \omega_{\mathrm{t} a}-\omega_{\mathrm{c} a}$ be the same for all the species. For all the $\omega_{a}^{2}$ 's thus defined to be positive (and to tend to zero, rendering the system free, when $\varpi \rightarrow 0$ ), all the $\omega_{\text {ca }}$ 's have to be positive, consequently the signs of all the charges have to be the same, such that $e_{a} B<0$. Then the LLL eigenfunctions of the Hamiltonian (6.6) satisfying the anyonic interchange conditions 四 have the form

$$
\tilde{\Psi}_{N}=\prod_{(a j)<(b k)}\left(z_{a j}-z_{b k}\right)^{\alpha_{a b}} \prod_{a}\left\{\prod_{j} z_{a j}^{\ell_{a j}}\right\}_{\mathrm{sym}}
$$

where $(a j)<(b k)$ means: $a<b$ or $(a=b$ and $j<k)$, so that each pair is counted only once; and symmetrization is performed over the coordinates of particles of the same species only. The energy of the state (6.7) is

$$
E_{\left\{\ell_{a j}\right\}}=\sum_{a} N_{a} \omega_{\mathrm{ta}}+\left[\sum_{a j} \ell_{a j}+\sum_{a} \frac{N_{a}\left(N_{a}-1\right)}{2} \alpha_{a a}+\frac{1}{2} \sum_{a b} N_{a} N_{b} \alpha_{a b}\right] \varpi .
$$

The LLL spectrum is obtained by letting the $\ell_{a j}$ 's run from 0 to $\infty$ with the restriction $\ell_{a j} \leq \ell_{a, j+1}$. The partition function is

$$
\begin{aligned}
Z_{N_{1} \ldots N_{s}}= & \exp \left[-\left(\sum_{a} \frac{N_{a}\left(N_{a}-1\right)}{2} \alpha_{a a}+\frac{1}{2} \sum_{a b} N_{a} N_{b} \alpha_{a b}\right) \beta \varpi\right] \\
& \times \prod_{a} \frac{e^{-N_{a} \beta \omega_{\mathrm{t} a}}}{\prod_{k=1}^{N_{a}}\left(1-e^{k \beta \varpi}\right)} .
\end{aligned}
$$




\subsection{Equation of state}

Let us now derive the equations of state for multispecies anyons in the LLL both in a (species-dependent, as explained above) harmonic potential and in a box (labeling the associated cluster and virial coefficients with superscripts $\omega$ and $V$, respectively).

The cluster expansion for the system in a harmonic potential reads

$$
\ln \Xi=\sum_{k_{1} \ldots k_{s}} b_{k_{1} \ldots k_{s}}^{\omega} z_{1}^{k_{1}} \cdots z_{s}^{k_{s}}
$$

where the cluster coefficients $b_{k_{1} \ldots k_{s}}^{\omega}$ can be expressed in terms of the partition functions in the standard way, by matching (6.10) to $\Xi=\sum_{k_{1} \ldots k_{s}} Z_{k_{1} \ldots k_{s}} z_{1}^{k_{1}} \cdots z_{s}^{k_{s}}$, and the partition functions are given by (6.9).

We are interested in going to the thermodynamic limit. In accordance with the aforesaid, the harmonic frequencies are $\omega_{a}^{2}=\left(\varpi+\omega_{\mathrm{c} a}\right)^{2}-\omega_{\mathrm{c} a}^{2}$, where $\omega_{\mathrm{c} a}=$ $-e_{a} B / 2 m_{a}$. The thermodynamic limit is understood as $\varpi \rightarrow 0$, so that $\omega_{a}^{2} \simeq$ $2 \varpi \omega_{\mathrm{c} a} \rightarrow 0$. To the leading order in $\beta \varpi$ and $\omega_{a} / \omega_{\mathrm{c} a}$, the cluster coefficients are

$$
b_{k_{1} \ldots k_{s}}^{\omega}=\left.\frac{e^{-\beta \sum_{a} k_{a} \omega_{c a}}}{\left(k_{1}+\cdots+k_{s}\right) \beta \varpi} f_{k_{1} \ldots k_{s}}\right|_{g_{a b} \rightarrow \alpha_{a b}} .
$$

Appearance of the coefficients $f_{k_{1} \ldots k_{s}}$ (2.13) in this expression indicates that exclusion statistics is present in the system. Noting that in the case at hand $Z_{1}^{\prime}(\beta)=1 / \beta \varpi$, we see that $Z_{1}^{\prime}(\beta)$ and $b_{k_{1} \ldots k_{s}}^{\omega}$ satisfy (3.4) and (3.5), respectively, with $\varepsilon_{a}^{(0)}=\omega_{\mathrm{c} a}$ and $\delta=0$ (constant density of states). We can thus conclude that the equation of state for LLL anyons in the harmonic potential considered coincides with (4.8), generalizing the result of Ref. 25] for a single species.

Now we turn to the equation of state for the same system in a box. There is a general procedure of deriving the equation of state in a box starting from the cluster coefficients in a harmonic potential (originally proposed for anyons [31] and then put on general grounds [32]). Here we follow the lines of Ref. [32]. Assuming that the external potential varies slowly in space, the relation $\ln \Xi=$ $\beta P V$ valid in a box is replaced by

$$
\ln \Xi=\beta \int d^{D} r P(\mathbf{r}),
$$

where $P(\mathbf{r})$ is the local pressure. The latter is evaluated within a small volume where the external potential $\Phi_{a}(\mathbf{r})$ acting on the particles of the $a$-th species is approximately constant, using the equation of state for particles in a box with the chemical potential $\mu_{a}$ replaced by $\mu_{a}-\Phi_{a}(\mathbf{r})$ :

$$
\beta P(\mathbf{r})=\frac{1}{V} \sum_{k_{1} \ldots k_{s}} b_{k_{1} \ldots k_{s}}^{V}\left(z_{1} e^{-\beta \Phi_{1}(\mathbf{r})}\right)^{k_{1}} \cdots\left(z_{s} e^{-\beta \Phi_{s}(\mathbf{r})}\right)^{k_{s}} .
$$

Substituting this into (6.12) and comparing the result to (6.10), one gets

$$
b_{k_{1} \ldots k_{s}}^{V}=V \frac{b_{k_{1} \ldots k_{s}}^{\omega}}{\int d^{D} r e^{-\beta \sum_{a} k_{a} \Phi_{a}(\mathbf{r})}} .
$$


For $D=2$ and $\Phi_{a}(\mathbf{r})=m_{a} \omega_{a} \mathbf{r}^{2} / 2$, this turns into $b_{k_{1} \ldots k_{s}}^{V}=\beta V\left(k_{1} m_{1} \omega_{1}^{2}+\right.$ $\left.\cdots+k_{s} m_{s} \omega_{s}^{2}\right) b_{k_{1} \ldots k_{s}}^{\omega} / 2 \pi$. In going to the thermodynamic limit, one has $m_{a} \omega_{a}^{2} \simeq$ $2 m_{a} \varpi \omega_{\mathrm{c} a}=2 \pi \varpi \rho_{\mathrm{L} a}$, where $\rho_{\mathrm{L} a}=m_{a} \omega_{\mathrm{c} a} / \pi$ is the Landau level degeneracy per unit area. Hence, we find the relation between the cluster coefficients of LLL anyons in a box and in a harmonic potential (the "thermodynamic limit prescription"):

$$
b_{k_{1} \ldots k_{s}}^{V}=V\left(k_{1} \rho_{\mathrm{L} 1}+\cdots+k_{s} \rho_{\mathrm{L} s}\right) \beta \varpi b_{k_{1} \ldots k_{s}}^{\omega} .
$$

Substituting (6.11), one finds

$$
b_{k_{1} \ldots k_{s}}^{V}=\left.V \frac{k_{1} \rho_{\mathrm{L} 1}+\cdots+k_{s} \rho_{\mathrm{L} s}}{k_{1}+\cdots+k_{s}} e^{-\beta \sum_{a} k_{a} \omega_{\mathrm{c} a}} f_{k_{1} \ldots k_{s}}\right|_{g_{a b} \rightarrow \alpha_{a b}}
$$

hence, the virial coefficients as in $(5.2)$ are

$$
a_{k_{1} \ldots k_{s}}=-\frac{\left(k_{1}+\cdots+k_{s}-1\right) !}{k_{1} ! \cdots k_{s} !} \sum_{a} \frac{\left[\left(\frac{\alpha_{a a}-1}{\alpha_{a a}}\right)^{k_{a}}-1\right] \prod_{b} \alpha_{a b}^{k_{b}}}{\rho_{\mathrm{L} a}^{k_{1}+\cdots+k_{s}-1}}
$$

which implies the following equation of state for LLL anyons in a box:

$$
\beta P=\sum_{a} \rho_{\mathrm{L} a} \ln \left(1+\frac{\rho_{a} / \rho_{\mathrm{L} a}}{1-\sum_{b} \alpha_{a b} \rho_{b} / \rho_{\mathrm{L} a}}\right) .
$$

This equation also shows that the statistical mechanics of the above system of anyons is governed by exclusion statistics. Indeed, starting directly from Haldane's definition (2.2) and assuming that all particles of the same species have the same energy $\varepsilon_{a}\left(\omega_{\mathrm{c} a}\right.$ in the case at hand), one easily derives (see also Ref. [6]) the equation of state which coincides with (6.18) upon changes $G_{a} / V \rightarrow \rho_{\mathrm{L} a}$ and $g_{a b} \rightarrow \alpha_{a b}$. [For a species-independent $\rho_{\mathrm{L} a} \equiv \rho_{\mathrm{L}}$, Eq. (6.16) is of course again a special case of (3.5), with $\varepsilon_{a}^{(0)}=\omega_{\mathrm{c} a}, Z_{1}^{\prime}(\beta)=\rho_{\mathrm{L}} V, \delta=-1$.]

It is possible to give a mean-field interpretation [21, 4] of Eq. (6.18) in the spirit of the Chern-Simons model. In that model, the statistics parameters are given by

$$
\alpha_{a b}=\frac{e_{a} \phi_{b}}{2 \pi}
$$

where $\phi_{b}$ is the Chern-Simons flux, whose proportionality to the charge $e_{b}$ ensures that $\alpha_{a b}$ is symmetric. Smearing out the fluxes and adding them to the external magnetic field changes the Landau density $\rho_{\mathrm{L} a}$ into $\rho_{\mathrm{L} a}^{\mathrm{eff}}=\rho_{\mathrm{L} a}-\sum_{b} \alpha_{a b} \rho_{b}$. Now, the quantity under the logarithm in (6.18) is $1+\rho_{a} / \rho_{\mathrm{L} a}^{\text {eff }}$, which is what one gets by setting all $\alpha_{a b}$ 's to zero and replacing $\rho_{\mathrm{L} a}$ with $\rho_{\mathrm{L} a}^{\mathrm{eff}}$. Equation (6.18) is different from the one conjectured previously in Ref. [21], although they coincide for a species-independent $\rho_{\mathrm{L}}$.

\footnotetext{
${ }^{5}$ Note that if the flux comes from a real solenoid, the corresponding formula is $\alpha_{a b}=$ $\left(e_{a} \phi_{b}+e_{b} \phi_{a}\right) / 2 \pi$, differing essentially by a factor of two from the above [33]. We thank Alfred Goldhaber for elucidating the point.
} 


\subsection{Semiclassical quantization}

Further insight into the nature of exclusion statistics in the system at hand can be gained by considering it semiclassically. The classical equation of motion of a particle in a magnetic field and a harmonic potential is, in our notation,

$$
\ddot{\mathbf{r}}=-\omega^{2} \mathbf{r}-2 \omega_{\mathrm{c}} \dot{\mathbf{r}} \times \mathbf{e}_{z}
$$

Normal modes, obviously, correspond to circles, with $\dot{\mathbf{r}} \times \mathbf{e}_{z}=-\Omega \mathbf{r}(e B<0$, so we have chosen positive $\Omega$ to correspond to clockwise rotation) and $\ddot{\mathbf{r}}=-\Omega^{2} \mathbf{r}$. Substituting into the above brings up two solutions,

$$
\Omega= \pm \omega_{\mathrm{t}}-\omega_{\mathrm{c}}
$$

with $\omega_{\mathrm{t}}$ defined as above [cf. (6.2)]. The upper sign corresponds to the lifted degeneracy of Landau levels, the lower one to Landau excitations; opposite signs of $\Omega$ mean that the directions of rotation corresponding to the two modes are opposite. The LLL restriction means that only the former, $\Omega=\varpi$ mode is excited, so that single-particle orbits are circles, the angular momentum is quantized in integer units, and the energy is

$$
E_{\ell}=\ell \varpi
$$

For a many-body system, the orbits are concentric circles and can be ordered by increasing value of angular momentum (or radius). Putting a flux $\phi$ inside the orbit of a charge $e$ increases the allowed values of the kinetic angular momentum by $\alpha=e \phi / 2 \pi$. Consequently, the quantization condition now is that the angular

momentum $\tilde{\ell}_{a j}$ of a particle is an integer plus additions from all particles whose orbits are inside (cf. [34):

$$
\tilde{\ell}_{a j}=\ell_{a j}+\sum_{b k} \alpha_{a b} \theta\left(\tilde{\ell}_{a j}-\tilde{\ell}_{b k}\right)
$$

[Eq. (6.19) has been taken into account], where $\ell_{a j}$ are integers and $\theta$ is the step function defined here as $\theta(x)=1$ for $x>0, \theta(x)=0$ for $x<0$, and $\theta(0)=1 / 2$. The total energy is $E=\sum_{a j} E_{\ell_{a j}}$, and substituting (6.22) and (6.23) herein yields the correct result (6.8), save for zero oscillations.

Eqs. (6.22)- 6.23) have recently appeared in Ref. [28] as defining exclusion statistics (in a somewhat different geometry); basically, they are the Bethe ansatz equations.

\section{Concluding remarks}

We have worked out in detail the statistical mechanics and thermodynamics for multispecies exclusion statistics starting from Haldane's combinatorial formula. As of now, two main groups of models are known to realize exclusion statistics: 
the two-dimensional LLL anyon model and one-dimensional integrable models. As for the first one, we have demonstrated that exchange statistics is equivalent to exclusion statistics if the charges of the particles are all of the same sign. The case when charges of both signs are present, which would be relevant, in particular, to quasielectrons and quasiholes in the FQHE, has yet to be investigated. In fact, the spectrum (6.8) does not lead to the thermodynamics considered for a nonsymmetric matrix $g_{a b}$, and it is unknown whether the LLL spectrum of anyons with different signs of charges does so.

Concerning integrable models, the most interesting question is as follows: What would be the generalization of the Calogero and Sutherland models that would realize exclusion statistics in the multispecies case? The virtual coincidence of the energy levels of single-species LLL anyons and of the Calogero model [21] suggests that the energy levels (6.8) for the multispecies case might at the same time be the energy levels of some integrable model. A similar argument applies to the Sutherland model, for which the generalized momenta $\ell$ would still satisfy an equation like (6.23), by the Bethe ansatz argument, but now with a quadratic dependence of the energy of free particles on those momenta.

One should note that different microscopic spectra may result in the same thermodynamics. In Ref. [35], a quantum mechanical spectrum for a singlespecies two-dimensional system was proposed which leads to an equation of state identical to that of two-dimensional exclusion statistics particles with quadratic dispersion (see Subsec. 5.1.3). The principal difference of our approach from that of Ref. [35] is that the spectrum (6.8) is derived from the rule of the kind (6.23) for the generalized ("renormalized" [36]) momenta (a generalization of the rule (6.23) can also be given for a two-dimensional system [30]). In terms of these momenta the energy has a form corresponding to a free system [cf. (6.22)], and the effect of statistics thus amounts to changing the bare momenta to their "renormalized" values determined by equations of the type (6.23), in accordance with the definition of multispecies exclusion statistics by the Bethe ansatz-like equations [28]. Along the above lines, the spectrum (6.8) and its two-dimensional generalization may be interpreted as the spectra of the systems discussed in Subsecs. 5.2.1 and 5.1.3, respectively [30].

As was recently discussed, the generalization of the Calogero-Sutherland model which keeps the interaction two-body and only allows different couplings between particles of different species implies constraints on possible values of the coupling parameters already at the level of the ground state of the system [37]. One might expect that the multispecies generalizations of the Calogero and Sutherland models which would reveal generic mutual exclusion statistics should involve three-body interactions, in the spirit of the approach of Ref. [38] which exploits analogies with anyons. 


\section{Acknowledgements}

We would like to thank Diptiman Sen for a discussion where he suggested that a system of anyons considered in Sec. 6 might be solvable. We wish to thank the Senter for Høyere Studier in Oslo, where this work was initiated, for kind hospitality and financial support. In deriving analytic expressions for coefficients of the expansions, Mathematica was used.

\section{References}

[1] F.D.M. Haldane, Phys. Rev. Lett. 67 (1991) 937.

[2] L. Brekke, A.F. Falk, S.J. Hugens, and T.D. Imbo, Phys. Lett. B 271 (1991) 73; A. Dasnières de Veigy and S. Ouvry, Phys. Lett. B 307 (1992) 91.

[3] F. Wilczek, Phys. Rev. Lett. 69 (1992) 132; Z.F. Ezawa and A. Iwazaki, Phys. Rev. B 47 (1993) 7295.

[4] S.B. Isakov, S. Mashkevich, and S. Ouvry, Nucl. Phys. B 448 (1995) 457.

[5] A. Dasnières de Veigy and S. Ouvry, Phys. Rev. Lett. 72 (1994) 600.

[6] Y.S. Wu, Phys. Rev. Lett. 73 (1994) 922.

[7] J.M. Leinaas and J. Myrheim, Phys. Rev. B 37 (1988) 9286; Int. J. Mod. Phys. B 5 (1991) 2573.

[8] T.H. Hansson, J.M. Leinaas, and J. Myrheim, Nucl. Phys. B 384 (1992) 559.

[9] A.P. Polychronakos, Nucl. Phys. B 324 (1989) 597.

[10] F. Calogero, J. Math. Phys. 10 (1969) 2191, 2197; 12 (1971) 419.

[11] B. Sutherland, J. Math. Phys. 12 (1971) 251.

[12] S.B. Isakov, Int. J. Mod. Phys. A 9 (1994) 2563.

[13] S.B. Isakov, Mod. Phys. Lett. B 8 (1994) 319.

[14] D. Bernard and Y.S. Wu, cond-mat/9404025.

[15] M.V.N. Murthy and R. Shankar, Phys. Rev. Lett. 73 (1994) 3331.

[16] Z.N.C. Ha, Nucl. Phys. B 435 (1995) 604.

[17] D.P. Arovas, J.R. Schrieffer, and F. Wilczek, Phys. Rev. Lett. 53 (1984) 722.

[18] M.D. Johnson and G.S. Canright, Phys. Rev. B 49 (1994) 2947; D. Li and S. Ouvry, Nucl. Phys. B 430 (1994) 563. 
[19] For a recent review, see e.g. X.-G. Wen, cond-mat/9506066.

[20] W.P. Su, Y.S. Wu, and J. Yang, Phys. Rev. Lett. 77 (1996) 3423; S.B. Isakov, G.S. Canright, and M.D. Johnson, Phys. Rev. B (to be published), condmat/9608139.

[21] A. Dasnières de Veigy and S. Ouvry, Mod. Phys. Lett. B 9 (1995) 271.

[22] C. Nayak, F. Wilczek, Phys. Rev. Lett. 73 (1994) 2740.

[23] S.B. Isakov, D.P. Arovas, J. Myrheim, and A.P. Polychronakos, Phys. Lett. A 212 (1996) 299.

[24] D. Sen and R.K. Bhaduri, Phys. Rev. Lett. 74 (1995) 3912.

[25] S.B. Isakov and S. Ouvry, J. Phys. A: Math. Gen. 29 (1996) 7401.

[26] T. Fukui and N. Kawakami, Phys. Rev. B 51 (1995) 5239.

[27] A. Dasnières de Veigy and S. Ouvry, Phys. Rev. Lett. 75 (1995) 352.

[28] S.B. Isakov and S. Viefers, Int. J. Mod. Phys. A (to be published), hepth/9605157.

[29] T.H. Hansson, J.M. Leinaas, and S. Viefers, Nucl. Phys. B 470 (1996) 291.

[30] S. Mashkevich, hep-th/9611048.

[31] A. Comtet, Y. Georgelin, and S. Ouvry, J. Phys. A: Math. Gen. 22 (1989) 3917.

[32] K. Olaussen, On the harmonic oscillator regularization of partition functions, Trondheim preprint No. 13 (1992), cond-mat/9207005.

[33] A.S. Goldhaber, R. MacKenzie, and F. Wilczek, Mod. Phys. Lett. A 4 (1989) 21.

[34] S. Kivelson, Phys. Rev. Lett. 65 (1990) 3369; J.Aa. Ruud and F. Ravndal, Phys. Lett. B 291 (1992) 137; N. Sivan and S. Levit, Phys. Rev. B 46 (1992) 3953.

[35] A. Dasnières de Veigy, Nucl. Phys. B 458 (1996) 533.

[36] N. Kawakami, J. Phys. Soc. Jpn. 62 (1993) 4163.

[37] D. Sen, Nucl. Phys. B 479 (1996) 554.

[38] C. Furtlehner and S. Ouvry, Mod. Phys. Lett. B 9 (1995) 503. 